\title{
Taxa de recuperação e características morfológicas de embriões muares (Equus caballus $x$ Equus asinus) ${ }^{1}$
}

\author{
Paula C.A. Silva ${ }^{2 *}$, Jhonnatha P. Oliveira², Gabriel A. Dutra², Sávio O. Paiva² \\ Diogo F. Caram², Renato G.C. Junqueira² e Julio C.F. Jacob²
}

\begin{abstract}
Silva P.C.A., Oliveira J.P., Dutra G.A., Paiva S.O., Caram D.F., Junqueira R.G.C. \& Jacob J.C.F. 2018. [Recovery rate and morphologic features of cross bred embryos (Equus caballus x Equus asinus).] Taxa de recuperação e características morfológicas de embriões muares (Equus caballus x Equus asinus). Pesquisa Veterinária Brasileira 38(7):1453-1457. Departamento de Reprodução e Avaliação Animal, Instituto de Zootecnia, Universidade Federal Rural do Rio de Janeiro, Seropédica, RJ 23890-000, Brazil. E-mail: paulinha_calmeida@hotmail.com

Production biotechnologies, particularly embryo transfer (ET) has constantly been contributed to reproduce horses and other species. The mules market has shown continuous growth, however, the biotechnology for mule assisted reproduction is still scarce. The aim of this study was to evaluate the embryo recovery rate and the features of the embryos from mares bred with donkeys. The embryos recovery attempts were performed on days 6 to 9 after ovulation, in order to evaluate the embryo recovery rate and the features related to age, morphology and embryonic diameter in each day. The overall embryo recovery rate was $55,9 \%(71 / 127)$, and there was no significant difference $(p>0,05)$ on different days (D6-D9). Embryos were recovered in stages of mórula, early blastocyst, blastocyst and expanded blastocyst. The diameter of the embryos ranged from $147-1688 \mu \mathrm{m}$ and the mean diameter of all the embryos collected was $438,04 \mu \mathrm{m}$. The collection of hybrid embryos might be performed between days 6 and 9 after ovulation, and provides recovery of embryos in the early stages of development.
\end{abstract}

INDEX TERMS: Recovery rate,morphology, cross bred, embryos, Equus caballus, Equus asinus, embryo transfer, hybrid, mare.

RESUMO.- A Transferência de Embrião (TE) contribuiu efetivamente para a produção de equinos e outras espécies. 0 mercado de muares tem apresentado um contínuo crescimento, entretanto, a aplicação das biotecnologias para a produção desses animais ainda é escassa. 0 presente estudo avaliou a taxa de recuperação embrionária e as características dos embriões provenientes do cruzamento de éguas com jumentos. Os embriões foram recuperados entre os dias 6 e 9 após a ovulação, dessa forma foi realizada a avaliação da taxa de recuperação embrionária e avaliação das características relacionadas com a idade, morfologia e diâmetro embrionário. A taxa de recuperação embrionária total foi de 55,9\% (71/127), e não apresentou diferença para as colheitas realizadas em diferentes dias (D6-D9). Foram recuperados embriões

\footnotetext{
${ }^{1}$ Recebido em 24 de maio de 2017.

Aceito ara publicação em 29 de junho de 2017.

${ }^{2}$ Departamento de Reprodução e Avaliação Animal, Universidade Federal Rural do Rio e Janeiro (UFRRJ), Rodovia BR-465 Km 7, Seropédica, RJ 23890-000, Brasil.*Autor para correspondência: paulinha_calmeida@hotmail.com
}

nos estágios de mórula, blastocisto inicial, blastocisto e blastocisto expandido. 0 tamanho dos embriões variou entre 147-1688 $\mu \mathrm{m}$ e a média do diâmetro de todos os embriões recuperados foi de $438,04 \mu \mathrm{m}$. A recuperação de embriões muares pode ser realizada entre os dias 6 e 9 após a ovulação, e propicia a recuperação de embriões nos primeiros estágios de desenvolvimento.

TERMOS DE INDEXAÇÃO: Recuperação, embrião,Equus caballus, Equus asinus, transferência de embrião, híbrido, égua, mula.

\section{INTRODUÇÃO}

Os muares são animais oriundos do cruzamento entre Equus asinus - asininos (jumento e jumenta) com equinos - Equus caballus (égua e garanhão), originando a mula, burro ou bardoto, dependendo do cruzamento realizado (Oliveira et al. 2007). Durante muito tempo os muares foram animais desvalorizados pelos criadores, atualmente as mulas e burros, são os híbridos mais conhecidos e utilizados no 
mundo (Gastal et al. 2014). Dessa maneira, o mercado de muares encontra-se em expansão e conta com animais de grande importância e valor zootécnico, mas, o número de mulas e burros ainda é pequeno em comparação ao número de equinos. Em equinos, a TE é uma técnica amplamente difundida e que permitiu a rápida expansão no número de animais (Squires et al. 2003, Lira et al. 2009, Bortot \& Zappa 2013), propiciou o conhecimento a respeito de características biológicas dos embriões como: diâmetro (Herrera et al. 2014), estágio (Choi et al. 2010, Panzani et al. 2014) e qualidade embrionária (McKinnon \& Squires 1988, McCue 2011), e possibilita seu uso juntamente com outras biotecnologias reprodutivas (McKinnon \& Squires 2007).

Em asininos a TE já foi utilizada algumas vezes, e os trabalhos reportam desde a taxa de recuperação para os diferentes dias de colheita (Camillo et al. 2010), bem como, as características envolvendo os embriões recuperados (Panzani et al. 2012) e ainda os fatores envolvendo as taxas de gestação pós-transferência (Allen et al. 1985, Vendramini et al. 1997). Quando se trata do uso da TE em muares, as publicações científicas referem-se ao uso das mulas como receptoras de embriões (Quintero et al. 1996), ou apresentam as divergências e problemas relacionadas com a gestação interespécie em éguas e jumentas (Allen \& Moor 1972, Allen 1975, Antczak et al. 1985, Allen et al. 1987, Bessent \& Ginther 1988, Gastal et al. 1993, Meira et al. 1998). Não há, contudo, dados publicados consistentes comparando taxa de recuperação, qualidade, diâmetro e estágio evolutivo embrionário, entre embriões equinos, muares e asininos.

0 presente estudo dedicou-se a avaliar a taxa de recuperação, diâmetro, qualidade, estágio e taxa de crescimento dos embriões provenientes do cruzamento de éguas com jumento, recuperados entre os dias 6 e 9 após a ovulação.

\section{MATERIAL E MÉTODOS}

A pesquisa foi realizada no Departamento de Reprodução e Avaliação Animal da UFRRJ, e foi aprovada pelo Comitê de Ética sob o número 23083.010030/2012-07.

Foram utilizados 127 ciclos estrais de 58 éguas, pertencentes as raças Bretão Postier, Mangalarga Marchador e Mestiças Mangalarga. As éguas entre3 e 15 anos apresentavam boa condição corporal com escore mínimo cinco (escala de classificação de um a nove) (Henneke et al. 1983), ciclos estrais regulares e histórico reprodutivo conhecido. 0 garanhão utilizado foi um jumento da raça Pêga, de fertilidade comprovada, que foi submentido a um exame andrológico prévio no início da estação de monta.

A atividade ovariana das éguas foi monitorada diariamente, por meio da palpação retal e técnica de ultrassonografia, com aparelho modelo Mindray DP2200Vet, equipado com transdutor linear de $5.0 \mathrm{MHz}$. Na presença de um folículo $>30 \mathrm{~mm}$ foi realizada a indução da ovulação, utilizando 1000UI (1,0 mL i.v.) de hCG - Gonadotrofina coriônica Humana (Chorulon ${ }^{\circledR}$ Intervet Schering-Plough Animal Health) ou 750 $\mu \mathrm{g}$ (3,0mLi.v.) de Acetato de Deslorelina (Sincrorrelin ${ }^{\circledR}$ - Ourofino Saúde Animal). As éguas foram inseminadas 24 horas após a indução da ovulação por via transcervical, com sêmen resfriado, diluído (Botu-Sêmen ${ }^{\circledR}$ Botupharma), na dose de 500 milhões de espermatozoides com movimento progressivo (70-80\%), vigor entre dois e três, e volume inseminante entre 10 e $15 \mathrm{~mL}$. Após a inseminação o acompanhamento folicular continuou diariamente até a detecção da ovulação, e nos casos em que esta não ocorreu em até 48 horas após a aplicação dos agentes indutores, uma nova inseminação foi realizada 48horas após a primeira inseminação artificial.

A colheita dos embriões foi realizada nos dias 6 (49 colheitas), 7 (49), 8 (24) e 9 (5), após ovulação das éguas, através da realização de lavado uterino transcervical (McKinnon \& Squires 1988). Após a colheita a primeira característica avaliada foi o diâmetro, que foi mensurado com o auxílio de uma ocular de 10x, com retículo micrométrico, acoplada ao microscópio esteroscópico (Tecnival CGA-6745). A mensuração do diâmetro (Fig.1) foi feita sempre sob o aumento de 40x, independente do dia de colheita, e transformados segundo a fórmula fornecida pelo fabricante: TEm = NDC x 0,19/(AO x ASZ), onde TEm = Tamanho do embrião que deseja determinar; NDC = número de divisões; $\mathrm{AO}=$ Ampliação da objetiva; e ASZ = Ampliação do sistema de zoom. Após a mensuração foi realizada a classificação dos embriões em relação ao estágio (Mórula - MO, Blastocisto Inicial - BI, Blastocisto - BL e Blastocisto Expandido- BX) e qualidade embrionária (grau I a IV), como descrito por McKinnon \& Squires (1988) para avaliação de embriões equinos.

Encerrado os procedimentos as éguas receberam uma aplicação intramuscular de 7,5mg de Dinoprost Trometamina - PGF2 $\alpha$ (Lutalyse ${ }^{\circledR}$ Zoetis-Pfizer) para encurtamento do diestro e indução de um novo ciclo estral.

Utilizando o Microsoft Office Excel, foi realizado o teste Exato de Fisher, com 5\% de significância para determinar a taxa de ovulação, recuperação embrionária para os diferentes dias de colheita, e os embriões de diferentes estágios. A regressão linear foi utilizada para verificar a taxa de crescimento diária dos embriões.

\section{RESULTADOS E DISCUSSÃO}

No presente estudo a ovulação ocorreu em até 48 horas em 80,3\% (102/127) das induções realizadas. Esse resultado corrobora com os resultados já descritos por outros autores que afirmam que se a indução da ovulação for realizada no momento adequado (presença de um folículo ovariano $>30 \mathrm{~mm}$ associado com edema uterino), a maioria das éguas irá ovular em até 48 horas após a aplicação dos hormônios, o que facilitao manejo no uso de diversas biotécnicas (Palmer 1993, Bergfelt 2000, Brinsko et al. 2000, Ginther et al. 2008, Samper 2008, Jacob et al. 2011).

O percentual de ciclos onde ocorreu a ovulação após 48 horas da aplicação dos fármacos foi de 81,4\% (57/70) e 78,9\% (45/57), parao hCG e Deslorelina, respectivamente. Não houve diferença entre $(p>0,05)$ entre os hormônios utilizados. Os resultados do presente estudo são compatíveis

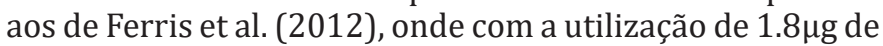

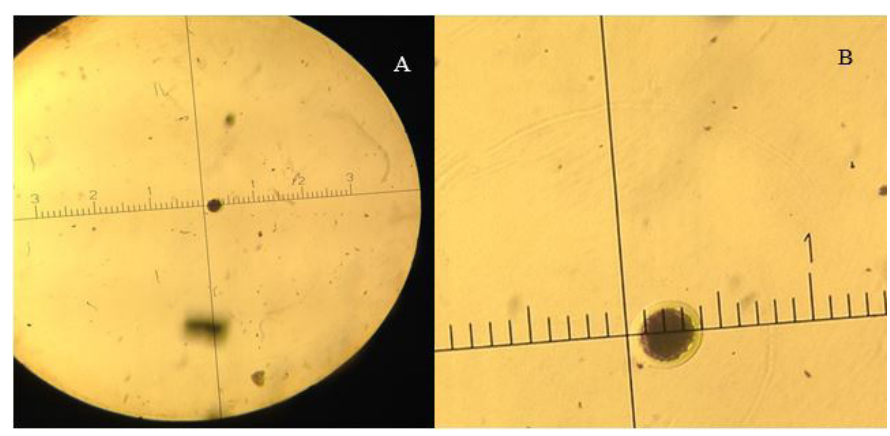

Fig.1. (A) Sistema micrométrico utilizado para mensurar o diâmetro dos embriões muares. (B) Aumento 400x utilizado para mensurar os embriões. 
Deslorelina e 2500UI de hCG o percentual de éguas ovuladas em até 48 horas da indução foi de 88,9\% (151/168) e 82,8\% (111/134), respectivamente. É importante ressaltar que no presente estudo as doses dos hormônios utilizados foram inferiores as doses do estudo citado, porém, o percentual de ovulações foi semelhante em ambos.

A colheita de embriões equinos, frequentemente é realizada entre 6 e 9 dias após a ovulação, entretanto, a taxa de recuperação embrionária no dia 6 é inferior aos demais dias (Squires \& Seidel 1995), no estudo em questão, a taxa de recuperação embrionária total foi de 55,9\% (71/127), e para os dias 6, 7, 8 e 9 após a ovulação, foi de 59,1\% (29/49), $49 \%(24 / 49), 62,5 \%(15 / 24), 60 \%(3 / 5)$, respectivamente (Quadro 1). Não houve diferença $(\mathrm{p}>0,05)$ entre os dias de colheita. Resultado diferente foi descrito por Jacob et al. (2010), onde a taxa de recuperação de embriões equinos no D6 $=42 \%(16 / 38)$, diferiu $(\mathrm{p}<0,03)$, das taxas no D7 $=61 \%$, $(159 / 262), \mathrm{D} 8=66 \%(285 / 434)$, D9 = 59\% (39/66) e D10 = 56\% (09/05). 0 mesmo foi observado por Camargo et al. (2013), onde no D6 $=18,1 \%(2 / 11)$ e D10 $=35,3 \%(6 / 17)$ as taxas de recuperação embrionária foram menores $(\mathrm{p}<0,01)$, que as observadas em D7 $=56 \%(14 / 25)$, D8 $=64,5 \%(189 / 293)$, e D9=52,6\% (181/344). Em jumentas, Camillo et al. (2010), também observaram diferenças na taxa de recuperação com uma menor taxa $(\mathrm{p}<0,05)$ no $\mathrm{D} 7=12,5 \%(1 / 8)$ em relação aos dias 8 e $9,79,1 \%$ (43/58) e 75\% (12/26), respectivamente. Os resultados do presente estudo nos fazem inferir que no dia 6, os embriões muares já se encontram todos no ambiente uterino, ao passo que os embriões equinos esse processo de migração inicia-se por volta do dia 5,5 e 6 (McKinnon \& Squires 2007).

Os embriões híbridos deste estudo foram identificados como: MO - mórula = 18,3\% (13/71); BI - blastocisto inicial = 26,8\% (19/71), BL - blastocisto=29,6\% (21/71), e BX- blastocisto expandido $=25,3 \%$ (18/71). De acordo com os diferentes dias de colheita foram identificados embriões em diferentes estágios de desenvolvimento onde D6 $=44,8 \%(13 / 29)$ MO e 55,2\% (16/29) BI, D7 = 12,5\% (3/24) BI, 62,5\% (15/24) BL e 25\% $(6 / 24)$ BX, D8 $=40 \%(6 / 15)$ BL e $60 \%(9 / 15)$ BX, e D9 $=100 \%$ (3/3) BX (Quadro 1). Em equinos os embriões recuperados, encontram-se nos estágios de MO (embrião de seis dias), BI (embriões de seis a sete dias), BL (embriões de sete dias) e BX (embriões de oito dias), entretanto, os embriões podem ser recuperados, em estágio incompatível com o dia de colheita, isso pode acontecer por fatores fisiológicos, como no caso de éguas idosas, onde ocorre um atraso no desenvolvimento, ou por falhas na detecção da ovulação (McKinnon \& Squires 1988). Em equinos as mórulas são difíceis de serem recuperadas, e ocorrem principalmente nas colheitas realizadas em D7 (Choi et al. 2010, Panzani et al. 2014). Neste estudo o número de mórulas recuperadas não diferiu dos demais estágios ( $p>0,05)$, e foram todas provenientes das colheitas realizadas 6 dias após a ovulação, sugerindo novamente que os embriões híbridos "cheguem" mais rápido ao útero do que os embriões equinos e asininos, sendo esse um ponto crucial na aplicação de biotecnologias que apresentam melhores resultados quando aplicadas em embriões em estágio inicial de desenvolvimento.

Dos embriões recuperados, 97,2\% (69/71) apresentaram morfologia compatível com grau de qualidade I, e 2,8\% (2/71) qualidade de grau II, segundo escala descrita por McKinnon \& Squires (1988) para embriões equinos. A qualidade dos embriões é um fator determinante nas taxas de prenhez. Embriões de qualidade inferior são provenientes de éguas mais velhas ou com problemas uterinos (Squires \& Seidel 1995, Panzani et al. 2012, Panzani et al. 2014).

O diâmetro dos embriões recuperados variou entre 147-1688 $\mu \mathrm{m}$, a média do diâmetro de todos os embriões recuperados foi de $438,04 \mu \mathrm{m}$, o tamanho médio (menor e maior diâmetro) dos embriões para os diferentes dias de colheita foi: D6 ( $n=29)-183,4 \mu m(147-253 \mu m), D 7(n=24)-463,2 \mu m$ $(168-886 \mu \mathrm{m}), \mathrm{D} 8(\mathrm{n}=15)-727,2 \mu \mathrm{m}(422-1224 \mu \mathrm{m}) \mathrm{e}$ D9 (n=3) - 1350,6 $\mu \mathrm{m}(844-1688 \mu \mathrm{m})$ (Quadro 1). 0 diâmetro dos embriões é uma característica muito importante uma vez que um embrião de diâmetro incompatível com a idade pode prejudicar a taxa de gestação. 0 diâmetro dos embriões híbridos corrobora com os achados referentes ao diâmetro dos embriões de éguas (Panzani et al. 2012)e jumentas (Panzani et al. 2014) para os diferentes dias de colheita. Através da regressão linear, identificou-se que os embriões apresentam um aumento médio no diâmetro de $312,7 \mu \mathrm{m}$ por dia após a ovulação, p<0,05(Fig.2).

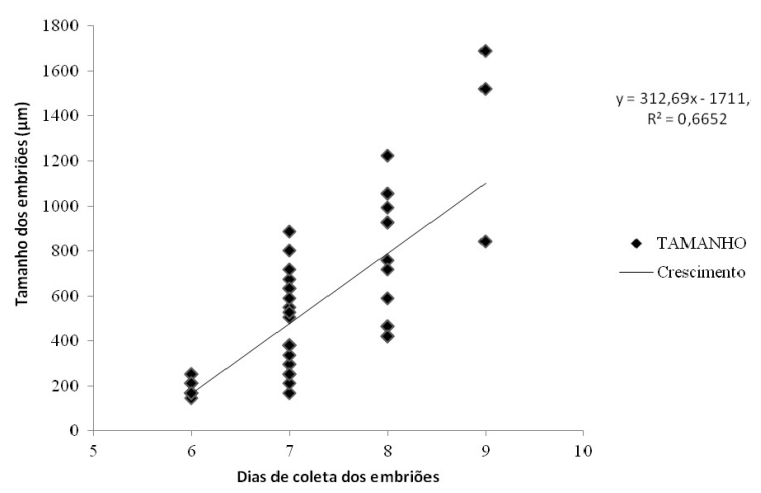

Fig.2. Diâmetro dos embriões muares $(\mathrm{n}=71)$ recuperados nos dias 6, 7, 8 e 9 após a ovulação, e taxa média de crescimento diário.

Quadro 1. Taxa de recuperação, estágios e diâmetro dos embriões muares recuperados nos diferentes dias de colheita

\begin{tabular}{|c|c|c|c|c|c|c|}
\hline \multirow{2}{*}{ Dia de colheita } & \multirow{2}{*}{$\begin{array}{c}\text { Taxa de } \\
\text { recuperação }\end{array}$} & \multicolumn{4}{|c|}{ Estágios } & \multirow{2}{*}{$\begin{array}{l}\text { Diâmetro médio } \\
\text { (mín - máx) }\end{array}$} \\
\hline & & MO & $\mathrm{BI}$ & $\mathrm{BL}$ & $\mathrm{BX}$ & \\
\hline D6 & $59,1 \%(29 / 49)$ & $44,8 \%(13 / 29)$ & $55,2 \%(16 / 29)$ & - & - & $183,4 \mu \mathrm{m}(147-253 \mu \mathrm{m})$ \\
\hline D7 & $49 \%(24 / 49)$ & - & $12,5 \%(3 / 24)$ & $62,5 \%(15 / 24)$ & $25 \%(6 / 24)$ & $463,2 \mu \mathrm{m}(168-886 \mu \mathrm{m})$ \\
\hline D9 & $60 \%(3 / 5)$ & - & - & - & $100 \%(3 / 3)$ & $1350,6 \mu \mathrm{m}(844-1688 \mu \mathrm{m})$ \\
\hline TOTAL & $55,9 \%(71 / 127)$ & $18,3 \%(13 / 71)$ & $26,8 \%(19 / 71)$ & $29,6 \%(21 / 71)$ & $25,3 \%(18 / 71)$ & $438,04 \mu \mathrm{m}(147-1688 \mu \mathrm{m})$ \\
\hline
\end{tabular}

D6 = Dia 6 após ovulação, D7 = Dia 7 após ovulação, D8 = Dia 8 após ovulação, D9 = Dia 9 após ovulação, MO = Mórula, BI = Blastocisto inicial, BL = Blastocisto, $\mathrm{BX}=$ Blastocisto expandido. 


\section{CONCLUSÕES}

A taxa de recuperação de embriões muares pós-inseminação de éguas com jumentos, é satisfatória e pode ser realizada entre os dias 6 e 9 pós-ovulação. A recuperação de embriões em estágio de mórula provenientes das colheitas realizadas no dia 6 evidencia uma possibilidade de precoce recuperação dos mesmos para sexagem, congelamento e outras biotécnicas.

A boa recuperação embrionária no D6 presume uma precocidade no processo de migração de embriões muares da tuba para o útero, fato que deve ser melhor elucidado.

Por fim, a despeito da aparente evolução mais rápida, embriões muares não diferem dos equinos e asininos em relação aos aspectos morfológicos.

Agradecimentos.- Ao Programa de Pós-graduação em Medicina Veterinária e à Coordenação de Aperfeiçoamento de Pessoal de Nível Superior (CAPES) pela concessão de bolsa e apoio financeiro na realização do trabalho.

\section{REFERÊNCIAS}

Allen W.R. 1975. The influence of fetal genotype upon endometrial cup development and PMSG and progestagen production in equids. J. Reprod. Fertility 23(23):405-413. <PMid:1060815>

Allen W.R. \& Moor R.M. 1972. The origin of the equine endometrial cups. I. Production of PMSG by fetal trophoblast cells. J. Reprod. Fertility 29(2):313316. <http://dx.doi.org/10.1530/jrf.0.0290313> <PMid:5023705>

Allen W.R., Kydd J., Boyle M.S. \& Antczac D.F. 1985. Between-species transfer of horse and donkey embryos: a valuable research tool. Equine Vet. J. 3:53-62.

Allen W.R., Kydd J.H., Boyle M.S. \& Antczak D.F. 1987. Extraspecific donkeyin-horse pregnancy as a model of early fetal death. J. Reprod. Fertility 35:197-209. <PMid:3479576>

Antczak D.F., Davles C.J., Kydd J.H. \& Allen W.R. 1985. Immunological aspects of pregnancy in mules. Equine Vet. J. 3:68-72.

Bergfelt D.R. 2000. Estrous synchronization. mare, p.195-228. In: Samper J.C. (Eds), Equine breeding management and artificial insemination. 2nd ed. Saunders, Philadelphia.

Bessent C. \& Ginther O.J. 1988. Comparison of early conceptus mobility between mares and jennies. Theriogenology 29(4):913-920. <http:// dx.doi.org/10.1016/0093-691X(88)90228-2><PMid:16726412>

Bortot D.C. \& Zappa V. 2013. Aspectos da reprodução equina: inseminação artificial e transferência de embrião: revisão de literatura. Revta Cient. Eletrôn. Med. Vet. 11(21):1-23.

Brinsko S., Varner D.D. \& Blanchard T.L. 2000. Transported equine sêmen, p.1-6. In: Ball B.A. (Eds), Recent Advances in Equine Reproduction. International Veterinary Information Service, Ithaca.

Camargo C.E., Weiss R.R., Kozicki L.E., Duarte M.P., Duarte M.C.G., Bertol M.A.F., Gaievski F.R. \& Bastos G.M. 2013. Aspectos relacionados com a recuperação embrionária em éguas da raça brasileiro de hipismo, utilizadas em um programa comercial de transferência de embrião. Vet. Zootec. 20:74-83.

Camillo F., Panzani D., Scollo A.C., Rota A., Crisci A., Vannozzi I. \& Balbo S. 2010. Embryo recovery rate and recipients' pregnancy rate after nonsurgical embryo transfer in donkeys. Theriogenology 73(7):959-965. <http:// dx.doi.org/10.1016/j.theriogenology.2009.11.024><PMid:20083302>

Choi Y.H., Gustafson-Seabury A., Velez I.C., Hartman D.L., Bliss S., Riera F.L., Roldán J.E., Chowdhary B. \& Hinrichs K. 2010. Viability of equine embryos after puncture of the capsule and biopsy for preimplantation genetic diagnosis. Reproduction 140(6):893-902.

Ferris R.A., Hatzel J.N., Lindholm A.R.G., Scofield D.B. \& Mccue P.M. 2012. Efiicacyof deslorelin acetate (sucromate) on induction of ovulation in
American quarter horse mares. J. Equine Vet. Sci. 32(5):285-288. <http:// dx.doi.org/10.1016/j.jevs.2011.11.007>

Gastal E.L., Santos G.F., Henry M. \& Piedade H.M. 1993. Embryonic and foetal development in donkeys. Equine Vet. J. 15:10-13.

Gastal E.L., Barros L.O., Carneiro G.F. \& Gastal M.O. 2014. Follicular dynamics in mules. J. Equine Vet. Sci. 34(1):144. <http://dx.doi.org/10.1016/j. jevs.2013.10.099>

Ginther O.J., Gastal E.L., Gastal M.O. \& Beg M.A. 2008. Dynamics of the equine preovulatory follicle and periovulatory hormones: what's new? J. Equine Vet. Sci. 28(8):454-460. <http://dx.doi.org/10.1016/j.jevs.2008.07.008>

Henneke D.R., Potter G.D., Kreider J.L. \& Yeates B.F. 1983. Relationship between body condition score, physical measurements and body fat percentage in mares. Equine Vet. J. 15(4):371-372. <http://dx.doi. org/10.1111/j.2042-3306.1983.tb01826.x><PMid:6641685>

Herrera C., Morikawa M.I., Bello M.B., von Meyeren M., Centeno J.E., Dufourq P., Martinez M.M. \& Llorente J. 2014. Setting up equine embryo gender determination by preimplantation genetic diagnosis in a commercial embryo transfer program. Theriogenology 81(5):758-763. <http://dx.doi. org/10.1016/j.theriogenology.2013.12.013><PMid:24439164>

Jacob J.C.F., Santos G.O., Sá M.A.F. \& Oliveira J.P. 2011. Uso clínico de hCG em um programa de transferência de embriões equino: mitos e verdades. Hora Vet. 30(180):9-13.

Jacob J.C.F., Santos G.O., Oliveira J.P., Gastal M.O. \& Gastal E.L. 2010. Evaluation of reproductive parameters in a commercial equine embryo transfer program. Anim. Reprod. Sci. 121(1/2):305-306. <http://dx.doi.org/10.1016/j. anireprosci.2010.04.174>

Lira R.A., Peixoto G.C.X. \& Silva A.R. 2009. Transferência de embrião em equinos: revisão. Acta Veterinaria Brasilica 3:132-140.

McCue P.M. 2011. Transferência de embriões em equinos: avaliação do embrião. Revta Educ. Contin. Med. Vet. Zootec. CRMV-SP. 9(3):80-83.

McKinnon A.O. \& Squires E.L. 1988. Equine embryo transfer. J. Am. Vet. Med. Assoc. 4(2):305-330. <PMid:3044542>

McKinnon A.O. \& Squires E.L. 2007.Embryo transfer and related technologies, p.319-334. In: Ibid. (Eds), Current Therapy Equine Reproduction. W.B. Saunders, St Louis, Missouri. <http://dx.doi.org/10.1016/B978-0-72160252-3.50055-2>.

Meira C., Ferreira J.C.P., Papa F.O. \& Henry M. 1998. Ultrasonographic evaluation of the conceptus from days 10 to 60 of pregnancy in jennies. Theriogenology 49(8):1475-1482. <http://dx.doi.org/10.1016/S0093691X(98)00093-4><PMid:10732011>

Oliveira V.B., Sanches S.B. \& Almeida F.Q. 2007. Muares: tema transversal para o ensino médio e técnico em Agropecuária. $2^{\text {a }}$ ed. Publit, Rio de Janeiro, p.13-14.

Palmer E. 1993. Induction of ovulation, p.344-347. In: McKinnon A.O. \& Voss J.L. (Eds), Equine Reproduction. Lea and Febiger, Malvern.

Panzani D., Rota A., Marmorini P., Vannozzi I. \& Camillo F. 2014. Retrospective study of factors affecting multiple ovulations, embryo recovery, quality, and diameter in a commercial equine embryo transfer program. Theriogenology 82(6):807-814.<http://dx.doi.org/10.1016/j.theriogenology.2014.06.020> $<$ PMid:25085596>

Panzani D., Rota A., Crisci A., Kindahl H., Govoni N. \& Camillo F. 2012. Embryo quality and transcervical technique are not limiting factors in donkey embryo transfer outcome. Theriogenology 77(3):563-569. <http://dx.doi. org/10.1016/j.theriogenology.2011.08.032><PMid:22056016>

Quintero F.M., Zarco L.Q. \& Valencia J.M. 1996. Transferencia de embriones híbridos (E. caballus x E. asinus) em mulas. Veterinaria México 27:175-177. 
Samper J.C. 2008. Induction of estrus and ovulation: why some mares respond and others do not. Theriogenology 70(3):445-447. <http:// dx.doi.org/10.1016/j.theriogenology.2008.04.040> <PMid:18554709>

Squires E.L. \& Seidel G.E. 1995. Collection and transfer of equine embryo. Anim. Reprod. Biotechnol. Lab. Bull. VIII, Colorado State University, Colorado, p.7-9.
Squires E.L., Carnevale E.M., McCue P.M. \& Bruemmer J.E. 2003. Embryo technologies in the horse. Theriogenology 59(1):151-170. <http://dx.doi. org/10.1016/S0093-691X(02)01268-2> <PMid:12499026>

Vendramini O.M., Bruyas J.F., Fieni F., Battut I. \& Tainturier D. 1997. Embryo transfer in Poitou donkeys: preliminary results. Theriogenology 47(1):409. 